\title{
Fermions and the scattering equations
}

\author{
Stefan Weinzierl \\ PRISMA Cluster of Excellence, Institut für Physik, Johannes Gutenberg-Universität Mainz, \\ D - 55099 Mainz, Germany \\ E-mail: stefanwethep.physik.uni-mainz.de
}

ABSTRACT: This paper investigates how tree-level amplitudes with massless quarks, gluons and/or massless scalars transforming under a single copy of the gauge group can be expressed in the context of the scattering equations as a sum over the inequivalent solutions of the scattering equations. In the case where the amplitudes satisfy cyclic invariance, $\mathrm{KK}$ - and $\mathrm{BCJ}$-relations the only modification is the generalisation of the permutation invariant function $E(z, p, \varepsilon)$. We present a method to compute the modified $\hat{E}(z, p, \varepsilon)$. The most important examples are tree amplitudes in $\mathcal{N}=4$ SYM and QCD amplitudes with one quark-antiquark pair and an arbitrary number of gluons. QCD amplitudes with two or more quark-antiquark pairs do not satisfy the BCJ-relations and require in addition a generalisation of the Parke-Taylor factors $C_{\sigma}(z)$. The simplest case of the QCD tree-level four-point amplitude with two quark-antiquark pairs is discussed explicitly.

KEYWORDS: Scattering Amplitudes, QCD

ARXIV EPRINT: 1412.5993 


\section{Contents}

1 Introduction 1

2 Review of basic facts 3

2.1 Colour decomposition 3

$\begin{array}{ll}2.2 \text { The scattering equations } & 6\end{array}$

$\begin{array}{lll}2.3 & \text { Amplitudes for scalars, gluons and gravitons } & 7\end{array}$

2.4 Relations among amplitudes 9

$\begin{array}{lll}2.5 & \text { KLT orthogonality } & 10\end{array}$

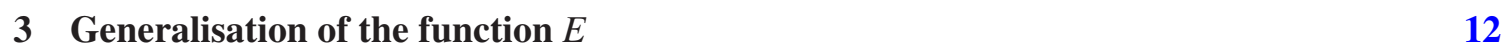

4 Generalisation of the Parke-Taylor factor $\quad 14$

5 Conclusions $\quad 16$

$\begin{array}{ll}\text { A Feynman rules } & 16\end{array}$

\section{Introduction}

The scattering equations are a set of algebraic equations, which associate to the $n$ momentum vectors of a scattering event $(n-3)$ ! inequivalent $n$-tuples of complex numbers $z=\left(z_{1}, \ldots, z_{n}\right)$. These scattering equations have been studied in a series of papers by Cachazo, He and Yuang [16]. It is remarkable, that tree amplitudes for scalars (spin 0), gluons (spin 1) or gravitons (spin 2) can all be expressed elegantly as a sum over the $(n-3)$ ! inequivalent solutions of the scattering equations. Hereby, the scalar theory consists of scalars charged under two gauge groups $\mathrm{U}(N)$ and $\mathrm{U}(\tilde{N})$ with cubic interactions. The full scalar amplitude is decomposed into "double-partial" amplitudes which are defined by a fixed colour order with respect to $\mathrm{U}(N)$ as well as $\mathrm{U}(\tilde{N})$. These double-partial amplitudes consist of Feynman diagrams compatible with both cyclic orders. The statement above refers to the double-partial amplitudes. In the gluon case it is well known that tree amplitudes can be decomposed into partial amplitudes with a fixed colour order (and hence with a fixed cyclic order). The statement above refers in the gluonic case to the partial amplitudes. Gravity amplitudes carry no additional gauge group information. Therefore they are not cyclic ordered. We see that while we increase the spin of the particles, the requirement on the cyclic ordering is relaxed.

The fact that tree amplitudes for scalars, gluons and gravitons can all be written as a sum over the solutions of the scattering equations has triggered significant interest in the community. Here, we list only a few examples: Dolan and Goddard [7] have provided a proof that the amplitudes can be written in this form. Their proof is based on Britto-Cachazo-Feng-Witten (BCFW) recursion relations [8]. In addition, they showed how to rewrite the scattering equations in polynomial 
form [9]. The solutions are thus a (zero-dimensional) algebraic variety. This idea has been generalised in [10]. There are close connections of the scattering equations with the construction of BCJ-numerators as shown in [11]. Analytic solutions of the scattering equations have been investigated in [12-14] and extensions to the scattering of massive particles are discussed in [7, 15]. In addition, there are interesting connections with string theory [16-21] and — of course - gravity [22-27].

As mentioned above, the formalism based on the scattering equations describes nicely the scattering of particles of spin 0,1 , and 2 . What is missing in this list are particles of half-integer spin, in particular fermions of spin $1 / 2$. Not much is known in this direction for non-supersymmetric theories. In supersymmetric theories amplitudes can be described in terms of superfields, and amplitudes with external fermions are just specific components of superamplitudes $[1,28]$. Furthermore, the four-fermion and the two-fermion-two-gluon amplitude were discussed explicitly in a supersymmetric string theory context [29].

In the pure gluonic case the scattering equations allow us to write the tree partial gluon amplitude as

$$
A_{n}(p, \varepsilon, \sigma)=i \sum_{\text {solutions } j} J\left(z^{(j)}, p\right) C_{\sigma}\left(z^{(j)}\right) E\left(z^{(j)}, p, \varepsilon\right)
$$

The exact definition of all quantities appearing in this equation will be given in section (2.3). The quantity $J$ is a Jacobian factor, depending on the variables of the scattering equations $z$ and the external momenta $p$. The Parke-Taylor factor $C_{\sigma}$ is a quantity depending only on $z$ and the cyclic order $\sigma$. The quantity $E$ depends on $z, p$ and the external polarisations $\varepsilon$, but does not depend on the cyclic order $\sigma$. Eq. (1.1) can be interpreted as a "factorisation of information": The information on the cyclic ordering is contained in $C_{\sigma}$, the information on the external polarisation is contained in $E$.

QCD amplitudes involving quarks can be decomposed into primitive amplitudes with a fixed cyclic ordering. The same can be done for amplitudes involving gluinos in $\mathcal{N}=4 \mathrm{SYM}$. In this paper we investigate cyclic ordered amplitudes with particles of spin up to 1 and study under which conditions a representation in the form as in eq. (1.1) with a modified function $\hat{E}$ exists. Our first assumption is that neither the scattering equations (and hence the Jacobian) nor the Parke-Taylor factor $C_{\sigma}$ are changed. We show that a representation in the form of eq. (1.1) with a modified $\hat{E}$ exists if and only if the amplitudes satisfy cyclic invariance, the Kleiss-Kuijf (KK) relations [30] and the Bern-Carrasco-Johansson (BCJ) relations [31]. The amplitudes may contain as external particles a combination of spin 1 , spin $1 / 2$ particles and spin 0 particles, all charged under a single gauge group. A typical example involving scalars are scalar particles in $\mathcal{N}=4 \mathrm{SYM}$. Note that the scalars in $\mathcal{N}=4 \mathrm{SYM}$ are not identical to the double-charged scalars mentioned earlier. In the case where a representation in the form of eq. (1.1) exists, we give a method to compute $\hat{E}$. Our method expresses $\hat{E}$ as a linear combination of $(n-3)$ ! basis amplitudes $A_{n}(\sigma)$. Tree amplitudes in any theory defined by a Lagrangian can be computed easily by a variety of methods (Feynman diagrams, Berends-Giele recursion relations [32], BCFW-recursion relations [8]). Therefore we can consider tree amplitudes to be known quantities. Our aim is not to find a computational method for an otherwise unknown quantity, in which case the approach of expressing $\hat{E}$ in terms of the quantities we would like to calculate would be tautological. The aim of this paper is to study 
whether a known quantity $A_{n}$ has a representation in the form of eq. (1.1) and to determine the function $\hat{E}$. The knowledge of the the function $\hat{E}$ is useful for generalised double-copy relations.

The conditions for such a representation to exist are that the amplitudes fulfil cyclic invariance, the KK-relations and the BCJ-relations. Tree amplitudes in $\mathcal{N}=4$ SYM satisfy these conditions and so do QCD amplitudes with a single quark-antiquark pair and an arbitrary number of gluons. However, although primitive QCD amplitudes with two or more quark-antiquark pairs satisfy cyclic invariance and the KK-relations, they do in general not satisfy the BCJ-relations. Hence, they do not have a representation in the form of eq. (1.1). ${ }^{1}$ The simplest example which does not have a representation in the form of eq. (1.1) is the QCD amplitude $A_{4}\left(\bar{q}, q, \bar{q}^{\prime}, q^{\prime}\right)$. The Parke-Taylor factor $C_{\sigma}$ encodes the set of relations consisting of cyclic invariance, KK-relations and BCJ-relations. Amplitudes not satisfying the BCJ-relations require apart from a modification of $E$ a generalisation of the Parke-Taylor factors $C_{\sigma}$. With the help of the pseudo-inverse of a matrix we derive a sufficient and necessary condition for a modified Parke-Taylor factor $\hat{C}_{\sigma}$. The simplest case of the QCD treelevel four-point amplitude $A_{4}\left(\bar{q}, q, \bar{q}^{\prime}, q^{\prime}\right)$ with two quark-antiquark pairs is worked out explicitly.

This paper is organised as follows: In section 2 we review basic facts about the colour decomposition of tree-level amplitudes, the scattering equations, relations satisfied by the amplitudes and the Kawai-Lewellen-Tye (KLT) orthogonality relations. In section 3 we present the generalisation for amplitudes satisfying cyclic invariance, KK- and BCJ-relations. In this case only a modification of the function $E(z, p, \varepsilon)$ is required. Section 4 is devoted to amplitudes not satisfying the BCJ-relations. These require in addition a modification of the Parke-Taylor factor $C_{\sigma}(z)$. Finally, section 5 contains the conclusions. For the convenience of the reader we included the cyclic ordered Feynman rules relevant to primitive amplitudes in a short appendix.

\section{Review of basic facts}

\subsection{Colour decomposition}

Amplitudes corresponding to theories with gauge groups may be decomposed into group-theoretical factors multiplied by kinematic functions called partial amplitudes. These partial amplitudes do not contain any colour information and are gauge invariant objects. Let us start the discussion with the case, where all particles belong to the adjoint representation of the gauge group. This is the case for amplitudes in $\mathcal{N}=4$ SYM and for gluon amplitudes in QCD. There are several possible choices for a basis in colour space. A possible choice is the colour-flow basis. This choice is obtained by attaching a factor

$$
\sqrt{2} T_{i j}^{a}
$$

to each external gluon and by using subsequently the Fierz identity to eliminate the adjoint indices. With the definition of the "colour closed strings"

$$
c_{\text {closed }}\left(g_{1}, \ldots, g_{n}\right)=\delta_{i_{1} j_{2}} \delta_{i_{2} j_{3}} \ldots \delta_{i_{n} j_{1}}
$$

we have the colour decomposition of the gluon tree amplitudes in QCD as

$$
\mathscr{A}_{n}^{\mathrm{QCD}}\left(g_{1}, g_{2}, \ldots, g_{n}\right)=\left(\frac{g}{\sqrt{2}}\right)^{n-2} \sum_{\sigma \in S_{n} / Z_{n}} c_{\text {closed }}\left(g_{\sigma_{1}}, \ldots, g_{\sigma_{n}}\right) A_{n}^{\text {partial }}\left(g_{\sigma_{1}}, \ldots, g_{\sigma_{n}}\right),
$$

\footnotetext{
${ }^{1}$ In the first version on the arxive it was erroneously claimed that they do satisfy the BCJ-relations.
} 
where the sum is over all non-cyclic permutations of the external gluon legs. The partial amplitudes $A_{n}^{\text {partial }}$ are gauge-invariant and have a fixed cyclic order. We note that theories with $\operatorname{SU}(N)$ gauge groups and $\mathrm{U}(N)$ gauge groups share the same partial amplitudes, since $\mathrm{U}(1)$-gluons cannot couple to particles in the adjoint representation. A similar decomposition as in eq. (2.3) exists for amplitudes $\mathscr{A}_{n}^{\text {SYM }}$ in $\mathcal{N}=4$ SYM.

Let us now turn to QCD tree amplitudes with quarks. We consider tree-level amplitudes with $n_{q}$ quarks, $n_{q}$ anti-quarks and $n_{g}$ gluons in an $\mathrm{SU}(N)$-gauge theory. The total number of external partons is therefore

$$
n=2 n_{q}+n_{g}
$$

All partons are assumed to be massless. We will call these amplitudes tree-level QCD amplitudes. Here, the decomposition into gauge-invariant cyclic ordered objects, which are called primitive tree amplitudes, is more involved and consists of three step: (i) elimination of identical flavours, (ii) stripping of colour factors, (iii) elimination of $\mathrm{U}(1)$-gluons.

(i) Elimination of identical flavours: Amplitudes with quark-antiquark pairs of identical flavour can always be related to amplitudes, where all quark-antiquark pairs have different flavours. This is achieved by summing over all quark permutations. An amplitude with $n_{q}$ quark-antiquark pairs can be written as

$$
\begin{aligned}
& \mathcal{A}_{n}^{\mathrm{QCD}}\left(\bar{q}_{1}, q_{1}, \ldots, \bar{q}_{2}, q_{2}, \ldots, \bar{q}_{n_{q}}, q_{n_{q}}\right)= \\
& \quad \sum_{\sigma \in S\left(n_{q}\right)}(-1)^{\sigma}\left(\prod_{j=1}^{n_{q}} \delta_{\bar{q}_{j} q_{\sigma(j)}}^{\mathrm{flav}}\right) \mathcal{A}_{n}^{\mathrm{non}-\mathrm{id}}\left(\bar{q}_{1}, q_{\sigma(1)}, \ldots, \bar{q}_{2}, q_{\sigma(2)}, \ldots, \bar{q}_{n_{q}}, q_{\sigma\left(n_{q}\right)}\right) .
\end{aligned}
$$

Here, $(-1)^{\sigma}$ equals -1 whenever the permutation is odd and equals +1 if the permutation is even. In $\mathcal{A}_{n}^{\text {non-id }}$ each external quark-antiquark pair $\left(\bar{q}_{j}, q_{\sigma(j)}\right)$ is connected by a continuous fermion line. The flavour factor $\delta_{\bar{q}_{j} q_{\sigma(j)}}^{\text {flav }}$ ensures that this combination is only taken into account, if $\bar{q}_{j}$ and $q_{\sigma(j)}$ have the same flavour. In $\mathcal{A}_{n}^{\text {non-id }}$ each external quark-antiquark pair $\left(\bar{q}_{j}, q_{\sigma(j)}\right)$ is treated as having a flavour different from all other quark-antiquark pairs. It is therefore sufficient to discuss only the case of different quark flavours and we will therefore from now on assume that all quark flavours are different.

(ii) Stripping of colour factors: As in the pure gluonic case we may decompose the amplitudes into group-theoretical factors (carrying the colour structures) multiplied by partial amplitudes. As before we work in the colour-flow basis and attach a factor $\sqrt{2} T_{i j}^{a}$ to each external gluon and use subsequently the $\mathrm{SU}(N)$-Fierz identity

$$
T_{i j}^{a} T_{k l}^{a}=\frac{1}{2}\left(\delta_{i l} \delta_{j k}-\frac{1}{N} \delta_{i j} \delta_{k l}\right) .
$$

to eliminate the adjoint indices. In addition to the "colour closed string" introduced in eq. (2.2) we also have to consider a "colour open string"

$$
c_{\text {open }}\left(q, g_{1}, \ldots, g_{n}, \bar{q}\right)=\delta_{i_{q} j_{1}} \delta_{i_{1} j_{2}} \ldots \delta_{i_{n} j_{\bar{q}}} .
$$

Born amplitudes with quarks have a decomposition in colour factors, which are products of colour open strings. The colour decomposition of a Born amplitude with $n_{q}$ quarks, $n_{q}$ antiquarks and $n_{g}$ 
gluons reads [33]

$$
\begin{aligned}
\mathcal{A}_{n}^{\text {non-id }}= & \left(\frac{g}{\sqrt{2}}\right)^{n-2} \sum_{\sigma \in S_{n_{g}}} \sum_{\pi \in S_{n_{q}}} \sum_{\substack{i_{1}, \ldots, i_{q} \geq 0 \\
i_{1}+\ldots+i_{n_{q}}=n_{g}}} c_{\mathrm{open}}\left(q_{1}, g_{\sigma_{1}}, \ldots, g_{\sigma_{i_{1}}}, \bar{q}_{\pi_{1}}\right) \\
& c_{\mathrm{open}}\left(q_{2}, g_{\sigma_{i_{1}+1}}, \ldots, g_{\sigma_{i_{1}+i_{2}}}, \bar{q}_{\pi_{2}}\right) \ldots c_{\mathrm{open}}\left(q_{n_{q}}, g_{\sigma_{i_{1}+\ldots+i_{n_{q}-1}+1}}, \ldots, g_{\sigma_{i_{1}+\ldots+i_{n q}}}, \bar{q}_{\pi_{n_{q}}}\right) \\
& A_{n}^{\text {partial }}\left(q_{1}, g_{\sigma_{1}}, \ldots, g_{\sigma_{i_{1}}}, \bar{q}_{\pi_{1}}, q_{2}, \ldots, g_{\sigma_{i_{1}+\ldots+i_{n} q}}, \bar{q}_{\pi_{n_{q}}}\right) .
\end{aligned}
$$

The sum over $\sigma$ is over all permutations of the external gluons, the sum over $\pi$ is over all permutations of the colour indices of the antiquarks. The sum over $\left\{i_{1}, \ldots, i_{n_{q}}\right\}$ is over all partitions of $n_{g}$ into $n_{q}$ non-negative integers and corresponds to the different possibilities to distribute $n_{g}$ gluons among $n_{q}$ open strings. The partial amplitudes $A_{n}^{\text {partial }}$ are gauge-invariant, but are in general for $n_{q}>2$ not cyclic ordered. This is related to the fact that for $n_{q} \geq 2$ there can be so-called $\mathrm{U}(1)$-gluons, corresponding to the second term of the Fierz identity in eq. (2.6).

(iii) Elimination of $\mathrm{U}(1)$-gluons: The partial amplitudes may be further decomposed into smaller objects, called primitive amplitudes. Primitive tree amplitudes are purely kinematic objects, which are gauge-invariant and which have a fixed cyclic ordering of the external legs. They are calculated from planar diagrams with the colour-ordered Feynman rules given in appendix A. These Feynman rules correspond to the colour-stripped Feynman rules of a $\mathrm{U}(N)$-gauge theory with quarks in the adjoint representation. In a $\mathrm{U}(N)$-gauge theory additional $\mathrm{U}(1)$-gluons are absent, since the Fierz identity for $\mathrm{U}(N)$ simply reads

$$
T_{i j}^{a} T_{k l}^{a}=\frac{1}{2} \delta_{i l} \delta_{j k}, \quad \text { for } \mathrm{U}(N) .
$$

With quarks in the adjoint representation, all colour-ordered three-valent vertices are anti-symmetric under the exchange of two of the three external particles. In general, the linear combination, which expresses a partial amplitude in terms of primitive amplitudes is not unique. This is due to the fact that there are in general relations among the primitive amplitudes. The Kleiss-Kuijf relations are an example. There is a systematic combinatorial algorithm for the decomposition of partial multi-quark amplitudes into primitive amplitudes, given in [34]. Any permutation may be written as a product of cycles. Let us assume that the permutation $\pi$ occurring in eq. (2.8) consists of $r$ cycles. We denote the order of the $i$-th cycle by $k_{i}$. Thus there are $k_{i}$ open strings associated to cycle $i$. Each cycle $i$ defines a cyclic word $u_{i}$, obtained by the concatenation of the arguments of the $k_{i}$ open strings. In this way we obtain $r$ cyclic words $u_{1}, \ldots, u_{r}$. Ref. [34] defines a shuffle operation $U\left(u_{1}, \ldots, u_{r}\right)$ and the combinatorial decomposition of the partial amplitude into primitive amplitudes is given by

$$
A_{n}^{\text {partial }}=\left(-\frac{1}{N}\right)^{r-1} \sum_{w \in U\left(u_{1}, \ldots, u_{r}\right)} A_{n}^{\text {primitive }}(w),
$$

where the sum is over all cyclic words appearing in the shuffle operation $U\left(u_{1}, \ldots, u_{r}\right)$.

For completeness we briefly discuss the colour decomposition of a cubic scalar theory with particles in the adjoint representation of $\mathrm{U}(N) \times \mathrm{U}(\tilde{N})$. Here we have a double decomposition with 
respect to the groups $\mathrm{U}(N)$ and $\mathrm{U}(\tilde{N})$ :

$$
\begin{aligned}
S_{n}\left(\phi_{1}, \phi_{2}, \ldots, \phi_{n}\right)= & \left(\frac{g}{2}\right)^{n-2} \sum_{\sigma, \tilde{\sigma} \in S_{n} / Z_{n}} c_{\text {closed }}\left(\phi_{\sigma_{1}}, \ldots, \phi_{\sigma_{n}}\right) c_{\text {closed }}\left(\phi_{\tilde{\sigma}_{1}}, \ldots, \phi_{\tilde{\sigma}_{n}}\right) \\
& S_{n}\left(\phi_{\sigma_{1}}, \ldots, \phi_{\sigma_{n}} \mid \phi_{\tilde{\sigma}_{1}}, \ldots, \phi_{\tilde{\sigma}_{n}}\right) .
\end{aligned}
$$

The partial amplitude $S_{n}\left(\phi_{\sigma_{1}}, \ldots, \phi_{\sigma_{n}} \mid \phi_{\tilde{\sigma}_{1}}, \ldots, \phi_{\tilde{\sigma}_{n}}\right)$ corresponds to Feynman diagrams compatible with the cyclic orders $\sigma$ and $\tilde{\sigma}$ [4].

\subsection{The scattering equations}

We denote by $\Phi_{n}$ the momentum configuration space of $n$ external massless particles:

$$
\Phi_{n}=\left\{\left(p_{1}, p_{2}, \ldots, p_{n}\right) \in(\mathbb{C} M)^{n} \mid p_{1}+p_{2}+\ldots+p_{n}=0, p_{1}^{2}=p_{2}^{2}=\ldots=p_{n}^{2}=0\right\} .
$$

In other words, a $n$-tuple $p=\left(p_{1}, p_{2}, \ldots, p_{n}\right)$ of momentum vectors belongs to $\Phi_{n}$ if this $n$-tuple satisfies momentum conservation and the mass-shell conditions $p_{i}^{2}=0$ for massless particles. It will be convenient to use the notation $p$ without any index to denote such an $n$-tuple.

We further denote by $\hat{\mathbb{C}}=\mathbb{C} \cup\{\infty\}$. The space $\hat{\mathbb{C}}$ is equivalent to the complex projective space $\mathbb{C P}^{1}$. For amplitudes with $n$ external particles we consider the space $\hat{\mathbb{C}}^{n}$. Points in $\hat{\mathbb{C}}^{n}$ will be denoted by $z=\left(z_{1}, z_{2}, \ldots, z_{n}\right)$. Again we use the convention that $z$ without any index denotes an $n$-tuple. We set for $1 \leq i \leq n$

$$
f_{i}(z, p)=\sum_{j=1, j \neq i}^{n} \frac{2 p_{i} \cdot p_{j}}{z_{i}-z_{j}}
$$

Differences like in the denominator will occur quite often in this article and we use the abbreviation

$$
z_{i j}=z_{i}-z_{j}
$$

The scattering equations read [3]

$$
f_{i}(z, p)=0
$$

For a fixed $p \in \Phi_{n}$ a solution of the scattering equation is a point $z \in \hat{\mathbb{C}}^{n}$, such that the scattering equations in eq. (2.15) are satisfied.

The scattering equations are invariant under the projective special linear group $\operatorname{PSL}(2, \mathbb{C})=$ $\operatorname{SL}(2, \mathbb{C}) / \mathbb{Z}_{2}$. Here, $\mathbb{Z}_{2}$ is given by $\{\mathbf{1},-\mathbf{1}\}$, with $\mathbf{1}$ denoting the $(2 \times 2)$-unit matrix. Let

$$
g=\left(\begin{array}{ll}
a & b \\
c & d
\end{array}\right) \in \operatorname{PSL}(2, \mathbb{C}) .
$$

Each $g \in \operatorname{PSL}(2, \mathbb{C})$ acts on a single $z_{i} \in \hat{\mathbb{C}}$ as follows:

$$
g \cdot z_{i}=\frac{a z_{i}+b}{c z_{i}+d}
$$

We further set

$$
g \cdot\left(z_{1}, z_{2}, \ldots, z_{n}\right)=\left(g \cdot z_{1}, g \cdot z_{2}, \ldots, g \cdot z_{n}\right)
$$


If $\left(z_{1}, z_{2}, \ldots, z_{n}\right)$ is a solution of eq. (2.15), then also $\left(z_{1}^{\prime}, z_{2}^{\prime}, \ldots, z_{n}^{\prime}\right)=g \cdot\left(z_{1}, z_{2}, \ldots, z_{n}\right)$ is a solution. We call two solutions which are related by a $\operatorname{PSL}(2, \mathbb{C})$-transformation equivalent solutions. We are in particular interested in the set of all inequivalent solutions of the scattering equations. As shown in $[1,2]$, there are $(n-3)$ ! different solutions not related by a $\operatorname{PSL}(2, \mathbb{C})$-transformation. We will denote a solution by

$$
z^{(j)}=\left(z_{1}^{(j)}, \ldots, z_{n}^{(j)}\right)
$$

and a sum over the $(n-3)$ ! inequivalent solutions by

$$
\sum_{\text {solution } j}
$$

The $n$ scattering equations in eq. (2.15) are not independent, only $(n-3)$ of them are. The Möbius invariance implies the relations

$$
\sum_{j=1}^{n} f_{j}(z, p)=0, \quad \sum_{j=1}^{n} z_{j} f_{j}(z, p)=0, \quad \sum_{j=1}^{n} z_{j}^{2} f_{j}(z, p)=0 .
$$

\subsection{Amplitudes for scalars, gluons and gravitons}

The scattering equations allow for an elegant representation of the scalar amplitude, the gluon amplitude and the graviton amplitude $[3,4,7]$. In this paragraph we review these representations. One first defines a $n \times n$-matrix $\Phi(z, p)$ with entries

$$
\Phi_{a b}(z, p)=\frac{\partial f_{a}(z, p)}{\partial z_{b}}=\left\{\begin{array}{cc}
\frac{2 p_{a} \cdot p_{b}}{z_{a b}^{2}} & a \neq b, \\
-\sum_{j=1, j \neq a}^{n} \frac{2 p_{a} \cdot p_{j}}{z_{a j}^{2}} & a=b .
\end{array}\right.
$$

Let $\Phi_{r s t}^{i j k}(z, p)$ denote the $(n-3) \times(n-3)$-matrix, where the rows $\{i, j, k\}$ and the columns $\{r, s, t\}$ have been deleted. We set

$$
\operatorname{det}^{\prime} \Phi(z, p)=(-1)^{i+j+k+r+s+t} \frac{\left|\Phi_{r s t}^{i j k}(z, p)\right|}{\left(z_{i j} z_{j k} z_{k i}\right)\left(z_{r s} z_{s t} z_{t r}\right)} .
$$

With the above sign included, the quantity $\operatorname{det}^{\prime} \Phi(z, p)$ is independent of the choice of $\{i, j, k\}$ and $\{r, s, t\}$. One defines a Jacobian factor by

$$
J(z, p)=\frac{1}{\operatorname{det}^{\prime} \Phi(z, p)} .
$$

One further defines a $(2 n) \times(2 n)$ antisymmetric matrix $\Psi(z, p, \varepsilon)$, where $\varepsilon=\left(\varepsilon_{1}, \ldots, \varepsilon_{n}\right)$ denotes the $n$-tuple of external polarisation vectors, through

$$
\Psi(z, p, \varepsilon)=\left(\begin{array}{cc}
A & -C^{T} \\
C & B
\end{array}\right)
$$

with

$$
A_{a b}=\left\{\begin{array}{cc}
\frac{2 p_{a} \cdot p_{b}}{z_{a b}} & a \neq b, \\
0 & a=b,
\end{array} \quad B_{a b}=\left\{\begin{array}{cc}
\frac{2 \varepsilon_{a} \cdot \varepsilon_{b}}{z_{a b}} & a \neq b \\
0 & a=b
\end{array}\right.\right.
$$


and

$$
C_{a b}=\left\{\begin{array}{cc}
\frac{2 \varepsilon_{a} \cdot p_{b}}{z_{a b}} & a \neq b, \\
-\sum_{j=1, j \neq a}^{n} \frac{2 \varepsilon_{a} \cdot p_{j}}{z_{a j}} & a=b .
\end{array}\right.
$$

For $1 \leq i<j \leq n$. one denotes by $\Psi_{i j}^{i j}(z, p, \varepsilon)$ the $(2 n-2) \times(2 n-2)$-matrix where the rows and columns $i$ and $j$ of $\Psi(z, p, \varepsilon)$ have been deleted. $\Psi_{i j}^{i j}(z, p, \varepsilon)$ has a non-vanishing Pfaffian, which is independent of $i$ and $j$, and one sets

$$
E(z, p, \varepsilon)=\frac{(-1)^{i+j}}{2 z_{i j}} \operatorname{Pf} \Psi_{i j}^{i j}(z, p, \varepsilon) .
$$

Under a $\operatorname{PSL}(2, \mathbb{C})$ transformation we have

$$
E(p, \varepsilon, g \cdot z)=\left(\prod_{j=1}^{n}\left(c z_{j}+d\right)^{2}\right) E(p, \varepsilon, z) .
$$

The function $E(z, p, \varepsilon)$ is gauge-invariant and all $z$-variables transform with weight 2 under $\operatorname{PSL}(2, \mathbb{C})$. However, when we expand the Pfaffian the individual terms in this expansion are in general neither gauge-invariant nor do they have the correct transformation properties with respect to $\operatorname{PSL}(2, \mathbb{C})$. We will later see that there is an expansion into a sum of terms, such that each term in this sum is gauge-invariant and has the correct $\operatorname{PSL}(2, \mathbb{C})$-transformation properties.

Let us now consider a permutation $\sigma \in S_{n}$. One defines a cyclic factor (or Parke-Taylor factor) by

$$
C_{\sigma}(z)=\frac{1}{z_{\sigma(1) \sigma(2)} z_{\sigma(2) \sigma(3)} \ldots z_{\sigma(n) \sigma(1)}} .
$$

Under PSL $(2, \mathbb{C})$ the Parke-Taylor factor transforms as

$$
C_{\sigma}(g \cdot z)=\left(\prod_{j=1}^{n}\left(c z_{j}+d\right)^{2}\right) C_{\sigma}(z) .
$$

We can now write the $n$-particle cyclic-ordered scalar amplitude $S_{n}$ with three-valent vertices, where we take for simplicity $\sigma=\tilde{\sigma}$ in eq. (2.11), the $n$-gluon partial amplitude $A_{n}$ and the $n$-graviton amplitude $M_{n}$ as $[3,4,7]$

$$
\begin{aligned}
S_{n}(p, \sigma) & =i \sum_{\text {solutions } j} J\left(z^{(j)}, p\right)\left[C_{\sigma}\left(z^{(j)}\right)\right]^{2} \\
A_{n}(p, \varepsilon, \sigma) & =i \sum_{\text {solutions } j} J\left(z^{(j)}, p\right) C_{\sigma}\left(z^{(j)}\right) E\left(z^{(j)}, p, \varepsilon\right), \\
M_{n}(p, \varepsilon) & =i \sum_{\text {solutions } j} J\left(z^{(j)}, p\right)\left[E\left(z^{(j)}, p, \varepsilon\right)\right]^{2} .
\end{aligned}
$$

We remark that the cyclic order $\sigma$ enters only through the cyclic factors $C_{\sigma}(z)$. The Jacobian $J(z, p)$ and the function $E(z, p, \varepsilon)$ are invariant under permutations. The polarisations of the external states are entirely contained in the function $E(z, p, \varepsilon)$.

Eq. (2.32) shows that the scattering equations can be used to express the scattering amplitudes of spin 0,1 , and 2 particles. This raises immediately the question, if also spin $1 / 2$ particles can be included in this representation. We will investigate this question in this paper. 


\subsection{Relations among amplitudes}

The pure gluonic tree amplitudes satisfy - as the tree amplitudes in $\mathcal{N}=4$ SYM - a set of equations, consisting of cyclic invariance relations, the Kleiss-Kuijf relations [30] and the BernCarrasco-Johansson relations [31]. This set of equations allow us to express any amplitude out of the $n$ ! possible external orderings as a linear combination of no more than $(n-3)$ ! orderings.

Clearly, cyclic ordered amplitudes are cyclic invariant. Cyclic invariance allows us to reduce the number of orderings from $n$ ! to $(n-1)$ ! and we may fix one external leg at a specific position, say external leg 1 at position 1 .

The Kleiss-Kuijf relations allow us to fix a second external leg at a second specified position, say external leg 2 at position 2 . This reduces the number of orderings to $(n-2)$ !. To state the Kleiss-Kuijf relations we let

$$
\vec{\alpha}=\left(\alpha_{1}, \ldots, \alpha_{j}\right), \vec{\beta}=\left(\beta_{1}, \ldots, \beta_{n-2-j}\right)
$$

and $\vec{\beta}^{T}=\left(\beta_{n-2-j}, \ldots, \beta_{1}\right)$. The Kleiss-Kuijf relations read

$$
A_{n}(1, \vec{\beta}, 2, \vec{\alpha})=(-1)^{n-2-j} \sum_{\sigma \in \vec{\alpha} \amalg \vec{\beta}^{T}} A_{n}\left(1,2, \sigma_{1}, \ldots, \sigma_{n-2}\right) .
$$

Here, $\vec{\alpha} \amalg \vec{\beta}^{T}$ denotes the set of all shuffles of $\vec{\alpha}$ with $\vec{\beta}^{T}$, i.e. the set of all permutations of the elements of $\vec{\alpha}$ and $\vec{\beta}^{T}$, which preserve the relative order of the elements of $\vec{\alpha}$ and of the elements of $\vec{\beta}^{T}$.

Finally, the BCJ relations allow to fix a third external leg at a third specified position, say external leg 3 at position 3 . This reduces the number of orderings to $(n-3)$ !. The $\mathrm{BCJ}$ relations have the form

$$
A_{n}(1,2, \vec{\beta}, 3, \vec{\alpha})=\sum_{\sigma \in P O P(\vec{\alpha}, \vec{\beta})} \mathcal{F}(\sigma) A_{n}\left(1,2,3, \sigma_{1}, \ldots, \sigma_{n-3}\right),
$$

where the sum is over all permutations of the set $\vec{\alpha} \cup \vec{\beta}$, which preserve the relative order of $\alpha$. The $\mathcal{F}(\sigma)$ are kinematical coefficients, given in [31].

A sufficient, but not necessary condition for amplitudes to satisfy the KK relations and the $\mathrm{BCJ}$ relations is the following criteria: If for all pairs $(i, j)$ there exists a BCFW-shift such that the amplitudes fall off like $1 / z$ if $i$ and $j$ are adjacent and like $1 / z^{2}$ if $i$ and $j$ are not adjacent, then the amplitudes satisfy the KK- and BCJ-relations. The proof of this statement can be found in [35]. This proof is entirely formulated in quantum field theory and uses only the Britto-Cachazo-FengWitten (BCFW) recursion relations [8]. It should be mentioned that historically the first proof of the $\mathrm{BCJ}$ relations has been derived from string theory [36, 37].

The pure gluonic tree amplitudes and amplitudes in $\mathcal{N}=4$ SYM have the required fall-off behaviour under BCFW-shifts [38-41] and therefore these amplitudes satisfy the KK- and BCJrelations [35, 42]. For amplitudes in $\mathcal{N}=4 \mathrm{SYM}$ it is essential that not only the (bosonic) momentum components but also the (fermionic) Grassmann components [39] are shifted. In this case the BCFW recursion relation relates an amplitude to lower-point amplitudes with different external states. 
The fact that the fall-off behaviour is sufficient but not necessary can be seen as follows: Let us consider a primitive QCD amplitude $A_{n}^{\text {primitive }}\left(\bar{q}_{1}, g_{2}, \ldots, g_{n-1}, q_{n}\right)$ with one quark-antiquark pair. Under a $\bar{q}_{1}-q_{n}$-shift the amplitude goes at the best to a constant for $z \rightarrow \infty$. However, the primitive amplitudes with one quark-antiquark pair and $(n-2)$-gluons satisfy the KK- and BCJ-relations. This follows from the fact, that these primitive amplitudes are identical to amplitudes with one gluino-antigluino pair and $(n-2)$ gluons in $\mathcal{N}=4$ SYM.

Now let us consider primitive QCD amplitudes $A_{n}^{\text {primitive }}$ with possibly multiple quark-antiquark pairs. These amplitudes satisfy the cyclic invariance relations and the KK-relations, but in general they do not satisfy the BCJ-relations. The proof that these amplitudes satisfy the KKrelations follows from the anti-symmetry of the vertices and can be found in [34]. In order to find a counter example of a primitive amplitude, which does not satisfy the BCJ-relations, we have to look at primitive amplitudes with at least two quark-antiquarks pairs. Primitive amplitudes with no quarks (i.e. the pure gluonic ones) as well as the amplitudes with exactly one quarkantiquark pair satisfy the BCJ-relations, as we have seen. The simplest counter example is given by $A_{4}^{\text {primitive }}\left(q_{1}, \bar{q}_{2}, q_{3}^{\prime}, \bar{q}_{4}^{\prime}\right)$. At four points the BCJ-relations read

$$
\begin{aligned}
& s_{23} A_{4}^{\text {primitive }}\left(g_{1}, g_{2}, g_{3}, g_{4}\right)=s_{24} A_{4}^{\text {primitive }}\left(g_{1}, g_{2}, g_{4}, g_{3}\right), \\
& s_{23} A_{4}^{\text {primitive }}\left(q_{1}, \bar{q}_{2}, g_{3}, g_{4}\right)=s_{24} A_{4}^{\text {primitive }}\left(q_{1}, \bar{q}_{2}, g_{4}, g_{3}\right),
\end{aligned}
$$

and hold for the $g_{1}, g_{2}, g_{3}, g_{4}$ and $q_{1}, \bar{q}_{2}, g_{3}, g_{4}$ amplitudes. Eq. (2.36) does not hold for the $q_{1}, \bar{q}_{2}$, $q_{3}^{\prime}, \bar{q}_{4}^{\prime}$ QCD amplitudes. Instead we have the (simpler) relation

$$
A_{4}^{\text {primitive }}\left(q_{1}, \bar{q}_{2}, q_{3}^{\prime}, \bar{q}_{4}^{\prime}\right)=-A_{4}^{\text {primitive }}\left(q_{1}, \bar{q}_{2}, \bar{q}_{4}^{\prime}, q_{3}^{\prime}\right) .
$$

The KK-relations imply then

$$
A_{4}^{\text {primitive }}\left(q_{1}, \bar{q}_{4}^{\prime}, \bar{q}_{2}, q_{3}^{\prime}\right)=0
$$

e.g. a primitive amplitude with crossed fermion lines vanishes.

We remark that the $\mathcal{N}=4$ SYM amplitude with two distinct gluino-antigluino pairs satisfies the BCJ-relations

$$
s_{23} A_{4}^{\mathrm{SYM}}\left(q_{1}, \bar{q}_{2}, q_{3}^{\prime}, \bar{q}_{4}^{\prime}\right)=s_{24} A_{4}^{\mathrm{SYM}}\left(q_{1}, \bar{q}_{2}, \bar{q}_{4}^{\prime}, q_{3}^{\prime}\right),
$$

as mentioned earlier. The amplitudes $A_{4}^{\text {primitive }}\left(q_{1}, \bar{q}_{2}, q_{3}^{\prime}, \bar{q}_{4}^{\prime}\right)$ and $A_{4}^{\mathrm{SYM}}\left(q_{1}, \bar{q}_{2}, q_{3}^{\prime}, \bar{q}_{4}^{\prime}\right)$ are not identical. The former is given by a single Feynman diagram consisting of a gluon exchange in the $s$-channel, while the latter is given by two diagrams, the first as above and the second given by a scalar exchange in the $t$-channel.

\subsection{KLT orthogonality}

We will need one more technical ingredient. The cyclic factors introduced in the previous paragraph satisfy an orthogonality relation called Kawai-Lewellen-Tye (KLT) orthogonality [2, 43-45].

We introduce first some notation: Let $\alpha \in S_{n}$ denote a permutation with

$$
\alpha(1)=1, \quad \alpha(n-1)=n-1, \quad \alpha(n)=n .
$$


We denote the subgroup of all such permutations with a slight abuse of notation by $S_{n-3}$. Similar, let $\beta \in S_{n}$ be a permutation with

$$
\beta(1)=1, \quad \beta(n-1)=n, \quad \beta(n)=n-1 .
$$

The subgroup of all such permutations is denoted by $\bar{S}_{n-3}$. Let $z^{(i)}=\left(z_{1}^{(i)}, z_{2}^{(i)}, \ldots, z_{n}^{(i)}\right)$ be a solution of the scattering equations. We write

$$
\begin{array}{ll}
C_{\alpha}^{i}=C_{\alpha}\left(z^{(i)}\right), & \alpha \in S_{n-3}, \\
\bar{C}_{\beta}^{i}=C_{\beta}\left(z^{(i)}\right), & \beta \in \bar{S}_{n-3} .
\end{array}
$$

For fixed $i$ we denote by $C^{i}$ the vector of all $C_{\alpha}^{i}$ with $\alpha \in S_{n-3}$. The vector $C^{i}$ has the dimension $(n-3)$ !, since there are $(n-3)$ ! elements in $S_{n-3}$. We have $(n-3)$ ! such vectors, since there are $(n-3)$ ! solutions of the scattering equations. We further denote by $\bar{C}^{j}$ the vector of all $C_{\beta}^{j}$ with $\beta \in \bar{S}_{n-3}$. The vector $\bar{C}^{j}$ has as well the dimension $(n-3)$ !, and there are again $(n-3)$ ! such vectors. An inner product is defined by

$$
\left(C^{i}, \bar{C}^{j}\right)=\sum_{\alpha \in S_{n-3}} \sum_{\beta \in \bar{S}_{n-3}} C_{\alpha}^{i} S[\alpha \mid \beta] \bar{C}_{\beta}^{j}
$$

with

$$
S[\alpha \mid \beta]=(-1)^{n} \prod_{i=2}^{n-2}\left(2 p_{1} \cdot p_{\alpha(i)}+\sum_{j=2}^{i-1} \theta_{\beta}(\alpha(j), \alpha(i)) 2 p_{\alpha(j)} \cdot p_{\alpha(i)}\right)
$$

and

$$
\theta_{\beta}(\alpha(j), \alpha(i))=\left\{\begin{array}{l}
1 \text { if } \alpha(j) \text { comes before } \alpha(i) \text { in the sequence }(\beta(2), \ldots, \beta(n-2)), \\
0 \text { otherwise. }
\end{array}\right.
$$

One has [2]

$$
\frac{\left(C^{i}, \bar{C}^{j}\right)}{\left(C^{i}, \bar{C}^{i}\right)^{\frac{1}{2}}\left(C^{j}, \bar{C}^{j}\right)^{\frac{1}{2}}}=\delta^{i j}
$$

and

$$
\left(C^{j}, \bar{C}^{j}\right)=\frac{1}{J\left(z^{(j)}, p\right)}
$$

Eq. (2.45) is the KLT orthogonality relation. Furthermore we have that the $(n-3) \times(n-3)$-matrix indexed by $\beta \in \bar{S}_{n-3}$ and $\gamma \in S_{n-3}$

$$
\sum_{\text {solutions } j} \bar{C}_{\beta}^{j} J\left(z^{(j)}, p\right) C_{\gamma}^{j}
$$

is an inverse to $S[\alpha \mid \beta]$, as shown in [4]:

$$
\sum_{\beta \in \bar{S}_{n-3}} S[\alpha \mid \beta] \sum_{\text {solutions } j} \bar{C}_{\beta}^{j} J\left(z^{(j)}, p\right) C_{\gamma}^{j}=\delta_{\alpha \gamma}
$$




\section{Generalisation of the function $E$}

Let us consider cyclic ordered amplitudes $A_{n}$, where the external particles have spin $\leq 1$. The external polarisations are now given by polarisation vectors $\varepsilon_{j}$ for external gluons, spinors $\bar{u}_{j}$ for out-going fermions, spinors $v_{j}$ for out-going anti-fermions and 1 for external scalars. For simplicity we will assume all particles to be out-going. In order to keep the notation simple we will continue to denote the set of external polarisations by $\varepsilon$. The cyclic order of the external particles will be denoted by $\sigma$. For $n$ external particles there are $n$ ! different cyclic orders.

We are interested in the question under which condition the amplitudes have a representation of the form as in eq. (1.1):

$$
A_{n}(p, \varepsilon, \sigma)=i \sum_{\text {solutions } j} J\left(z^{(j)}, p\right) C_{\sigma}\left(z^{(j)}\right) \hat{E}\left(z^{(j)}, p, \varepsilon\right) .
$$

Here, the Jacobian $J$ and the cyclic factor $C_{\sigma}$ are identical to eq. (2.24) and eq. (2.30), respectively. We only allow at this stage a modification of the function $E$. If such a representation exists, we would like to know $\hat{E}\left(z^{(j)}, p, \varepsilon\right)$.

We have the following theorem: For a given set of external particles with spin $\leq 1$ a representation in the form of eq. (3.1) exists if and only if the amplitudes with this set of external particles satisfy cyclic invariance, the KK-relations and the BCJ-relations. In this case the modified function $\hat{E}\left(z^{(j)}, p, \varepsilon\right)$ is given by

$$
\hat{E}\left(z^{(j)}, p, \varepsilon\right)=-i \sum_{\alpha \in S_{n-3}} \sum_{\beta \in \bar{S}_{n-3}} A_{n}(p, \varepsilon, \alpha) S[\alpha \mid \beta] \bar{C}_{\beta}^{j} .
$$

Note that each term in eq. (3.2) is gauge invariant and has the correct transformation properties under $\operatorname{PSL}(2, \mathbb{C})$. As mentioned in the introduction we consider tree amplitudes in any theory defined by a Lagrangian to be known quantities, which can be computed by a variety of methods (Feynman diagrams, Berends-Giele recursion relations [32], BCFW-recursion relations [8], etc.). Therefore $\hat{E}\left(z^{(j)}, p, \varepsilon\right)$ can be computed as well. Let us emphasize that the goal is not to find a new stand-alone method to compute $A_{n}$, but rather to investigate under which condition a representation in the form of eq. (3.1) exists, and in the case it does, to find a method to compute $\hat{E}\left(z^{(j)}, p, \varepsilon\right)$.

In order to prove the theorem let us first assume that the amplitudes $A_{n}$ satisfy cyclic invariance, KK-relations and BCJ-relations. Let us define quantities $\hat{A}_{n}$ by

$$
\hat{A}_{n}(p, \varepsilon, \sigma)=i \sum_{\text {solutions } j} J\left(z^{(j)}, p\right) C_{\sigma}\left(z^{(j)}\right) \hat{E}\left(z^{(j)}, p, \varepsilon\right),
$$

with $\hat{E}\left(z^{(j)}, p, \varepsilon\right)$ given by eq. (3.2). We have to show that

$$
\hat{A}_{n}(p, \varepsilon, \sigma)=A_{n}(p, \varepsilon, \sigma)
$$

for all $\sigma \in S_{n}$.

Eq. (3.4) is easily proven for $\sigma \in S_{n-3}$ : We have with eq. (2.48)

$$
\begin{aligned}
\hat{A}_{n}(p, \varepsilon, \sigma) & =\sum_{\text {solutions } j} \sum_{\alpha \in S_{n-3}} \sum_{\beta \in \bar{S}_{n-3}} A_{n}(p, \varepsilon, \alpha) S[\alpha \mid \beta] \bar{C}_{\beta}^{j} J\left(z^{(j)}, p\right) C_{\sigma}^{j} \\
& =\sum_{\alpha \in S_{n-3}} A_{n}(p, \varepsilon, \alpha) \delta_{\alpha \sigma} \\
& =A_{n}(p, \varepsilon, \sigma) .
\end{aligned}
$$


In order to prove eq. (3.4) for $\sigma \in S_{n} \backslash S_{n-3}$ it is the easiest to show that eq. (3.1) satisfies cyclic invariance, the Kleiss-Kuijf-relations and the Bern-Carrasco-Johansson relations. These relations allow to express any of $n$ ! possible external orderings in a basis of $(n-3)$ ! orderings. As such a basis we can take the $(n-3)$ ! orderings, for which we have already proven our claim in eq. (3.5).

The cyclic invariance is clear from an inspection of eq. (3.1). The cyclic order $\sigma$ enters only through $C_{\sigma}^{j}$, which is cyclic invariant.

The Kleiss-Kuijf relations read (we drop the arguments $p$ and $\varepsilon$ for the amplitudes)

$$
\hat{A}_{n}(1, \vec{\beta}, 2, \vec{\alpha})=(-1)^{n-2-j} \sum_{\sigma \in \vec{\alpha} \amalg \vec{\beta}^{T}} \hat{A}_{n}\left(1,2, \sigma_{1}, \ldots, \sigma_{n-2}\right) .
$$

In our case this reduces to an algebraic relation between the cyclic factors $C_{\sigma}^{j}$

$$
C_{(1, \vec{\beta}, 2, \vec{\alpha})}^{j}=(-1)^{n-2-j} \sum_{\sigma \in \vec{\alpha} \amalg \vec{\beta}^{T}} C_{\left(1,2, \sigma_{1}, \ldots, \sigma_{n-2}\right)}^{j},
$$

which is again easily verified. We remark that eq. (3.7) holds independently of $z^{(j)}$ being a solution of the scattering equations or not.

The BCJ relations reduce as well to a relation for the cyclic factors $C_{\sigma}^{j}$ :

$$
C_{(1,2, \vec{\beta}, 3, \vec{\alpha})}^{j}=\sum_{\sigma \in P O P(\vec{\alpha}, \vec{\beta})} \mathcal{F}(\sigma) C_{\left(1,2,3, \sigma_{1}, \ldots, \sigma_{n-3}\right)}^{j},
$$

We now need that $z^{(j)}$ is a solution of the scattering equations. However, eq. (3.8) is independent of the flavour of the external particles and its validity can be inferred from the pure gluonic case. Therefore we have shown $\hat{A}_{n}(p, \varepsilon, \sigma)=A_{n}(p, \varepsilon, \sigma)$ for all $\sigma \in S_{n}$.

Now let us assume that the amplitudes $A_{n}$ do not satisfy the set of relations given by cyclic invariance, KK-relations and BCJ-relations. This means that there is at least one relation from this set, which is violated. Let us write this relation as

$$
\sum_{\sigma \in S_{n}} R(\sigma) A_{n}(p, \varepsilon, \sigma) \neq 0
$$

Now let us further assume that $A_{n}$ has a representation in the form of eq. (3.1). In this representation the dependence on the cyclic order enters only through the Parke-Taylor factors $C_{\sigma}^{j}$. Any quantity, which has a representation of this form satisfies cyclic invariance, KK-relations and BCJ-relations. Therefore

$$
\sum_{\sigma \in S_{n}} R(\sigma) A_{n}(p, \varepsilon, \sigma)=0
$$

which is a contradiction to eq. (3.9). Therefore a representation of this form cannot exist. This completes the proof of the theorem.

Important examples, where a representation in the form of eq. (3.1) exists, are - apart from the pure gluonic tree amplitudes - all tree amplitudes in $\mathcal{N}=4$ SYM and the QCD tree amplitudes with one quark-antiquark pair and an arbitrary number of gluons. In all these cases, the function $\hat{E}\left(z^{(j)}, p, \varepsilon\right)$ is given by eq. (3.2). 
Let us briefly consider MHV amplitudes with one quark-antiquark pair

$$
A_{n}\left(q_{1}^{+}, g_{2}^{+}, \ldots, g_{l}^{-}, \ldots, g_{n-1}^{+}, \bar{q}_{n}^{-}\right)
$$

There is a SUSY Ward identity, which relates this amplitude to the corresponding all-gluon MHV amplitude:

$$
A_{n}\left(q_{1}^{+}, g_{2}^{+}, \ldots, g_{l}^{-}, \ldots, g_{n-1}^{+}, \bar{q}_{n}^{-}\right)=-\frac{\langle l 1\rangle}{\langle l n\rangle} A_{n}\left(g_{1}^{+}, g_{2}^{+}, \ldots, g_{l}^{-}, \ldots, g_{n-1}^{+}, g_{n}^{-}\right) .
$$

It follows that the function $\hat{E}\left(z^{(j)}, p, \varepsilon\right)$ is given for the $q_{1}^{+}, g_{2}^{+}, \ldots, g_{l}^{-}, \ldots, g_{n-1}^{+}, \bar{q}_{n}^{-}$-case on the scattering variety by

$$
\hat{E}\left(z^{(j)}, p, \varepsilon\right)=-\frac{\langle l 1\rangle}{\langle\ln \rangle} \frac{(-1)^{a+b}}{2 z_{a b}^{(j)}} \operatorname{Pf} \Psi_{a b}^{a b}\left(z^{(j)}, p, \varepsilon\right),
$$

with $\Psi$ defined by eqs. (2.25)-(2.27). Note that in $\Psi$ all external polarisations are gluon polarisation vectors.

\section{Generalisation of the Parke-Taylor factor}

In the previous section we gave a sufficient and necessary condition for a set of amplitudes to have a representation of the form (3.1). This applies to the pure gluonic amplitudes, all amplitudes in $\mathcal{N}=4$ SYM and the QCD tree amplitudes with one quark-antiquark pair and an arbitrary number of gluons. The most notable exceptions are the QCD primitive tree amplitudes with two or more quark-antiquark pairs. These amplitudes do in general not satisfy the BCJ relations. The simplest example of such an exception is the four-point amplitude $A_{4}^{\text {primitive }}\left(q_{1}, \bar{q}_{2}, q_{3}^{\prime}, \bar{q}_{4}^{\prime}\right)$. In order to present these amplitudes as a sum over the solution of the scattering equation we will need a further generalisation of eq. (1.1). Since the Parke-Taylor factors $C_{\sigma}$ have the set of cyclic invariance, KK- and BCJ-relations buried in them, we have to look at generalisations of the Parke-Taylor factors. Let us denote the information on the flavours of the external particles collectively by $f$. We consider a representation of the form

$$
A_{n}(p, \varepsilon, \sigma)=i \sum_{\text {solutions } j} J\left(z^{(j)}, p\right) \hat{C}_{\sigma}\left(z^{(j)}, f\right) \hat{E}\left(z^{(j)}, p, \varepsilon\right) .
$$

Note that this form keeps as much as possible the original structure and can again be interpreted as a "factorisation of information": The information on the external polarisations enters only through $\hat{E}$, the information on the cyclic order only through $\hat{C}$. The information on the flavours of the external particles enters $\hat{E}$ and $\hat{C}$. The Jacobian $J$ is defined as before. We will first investigate under which conditions such a representation may exist. In order to simplify the discussion we introduce the following short-hand notation: We define a $n$ !-dimensional vector $A_{\sigma}$ with components

$$
A_{\sigma}=A_{n}(p, \varepsilon, \sigma),
$$

a $n ! \times(n-3)$ !-dimensional matrix $M_{\sigma j}$ by

$$
M_{\sigma j}=J\left(z^{(j)}, p\right) \hat{C}_{\sigma}\left(z^{(j)}, f\right),
$$


and a $(n-3)$ !-dimensional vector $\hat{E}_{j}$ by

$$
\hat{E}_{j}=\hat{E}\left(z^{(j)}, p, \varepsilon\right) .
$$

Then eq. (4.1) may be written compactly as

$$
A_{\sigma}=i M_{\sigma j} \hat{E}_{j},
$$

where a sum over $j$ is understood. Let us further denote a pseudo-inverse matrix of $M_{\sigma j}$ by $N_{j \sigma}$. $N_{j \sigma}$ is a $(n-3) ! \times n !$-dimensional matrix with the property

$$
M N M=M, N M N=N .
$$

From the theory of pseudo-inverse matrices $[46,47]$ we have the following statement: A representation in the form of eq. (4.1) exists if and only if

$$
M_{\sigma j} N_{j \tau} A_{\tau}=A_{\sigma},
$$

in other words $A_{\sigma}$ must be an eigenvector of $M N$ with eigenvalue 1 . In this case $\hat{E}_{j}$ is given by

$$
\hat{E}_{j}=-i N_{j \sigma} A_{\sigma}-i\left(\delta_{j k}-N_{j \tau} M_{\tau k}\right) u_{k},
$$

where $u_{k}$ is an arbitrary $(n-3)$ !-dimensional vector. In the case, where $M_{\sigma j}$ has rank $(n-3)$ !, a pseudo-inverse is given by

$$
N=\left(M^{T} M\right)^{-1} M^{T}
$$

and we further have $N_{j \tau} M_{\tau k}=\delta_{j k}$. Then eq. (4.8) reduces to

$$
\hat{E}_{j}=-i N_{j \sigma} A_{\sigma} .
$$

eq. (4.7) allows us to verify or falsify an ansatz for $\hat{C}_{\sigma}$. With a valid ansatz for $\hat{C}_{\sigma}$, the function $\hat{E}$ is then given by eq. (4.8) or eq. (4.10).

Let us now look at the example $A_{4}^{\text {primitive }}\left(q_{1}, \bar{q}_{2}, q_{3}^{\prime}, \bar{q}_{4}^{\prime}\right)$. We set

$$
\hat{C}_{\sigma}(z, f)=\left\{\begin{aligned}
\frac{1}{z_{12} z_{23} z_{33} z_{41}}, & \text { if the cyclic order is } q_{1}, \bar{q}_{2}, q_{3}^{\prime}, \bar{q}_{4}^{\prime}, \\
-\frac{1}{z_{12} z_{23} z_{34} z_{41}}, & \text { if the cyclic order is } q_{1}, \bar{q}_{2}, \bar{q}_{4}^{\prime}, q_{3}^{\prime}, \\
0, & \text { if the cyclic order is } q_{1}, q_{3}^{\prime}, \bar{q}_{2}, \bar{q}_{4}^{\prime} .
\end{aligned}\right.
$$

With

$$
\hat{E}(z, p, \varepsilon)=-i \frac{1}{z_{12} z_{24} z_{43} z_{31}} s_{12} A_{4}^{\text {primitive }}\left(q_{1}, \bar{q}_{2}, q_{3}^{\prime}, \bar{q}_{4}^{\prime}\right)
$$

we then have

$$
A_{4}^{\text {primitive }}(p, \varepsilon, \sigma)=i \sum_{\text {solutions } j} J\left(z^{(j)}, p\right) \hat{C}_{\sigma}\left(z^{(j)}, f\right) \hat{E}\left(z^{(j)}, p, \varepsilon\right)
$$

for all $\sigma \in S_{4}$. 


\section{Conclusions}

In this paper we discussed the extension of the representation of a cyclic ordered tree amplitude in the framework of the scattering equations from the pure gluonic case to the case, where the external particles are allowed to have spin $\leq 1$. In the case where the amplitudes satisfy cyclic invariance, the KK- and BCJ-relations the only required modification is the generalisation of the permutation invariant function $E(z, p, \varepsilon)$. The most important examples are tree amplitudes in $\mathcal{N}=4 \mathrm{SYM}$ and QCD amplitudes with one quark-antiquark pair and an arbitrary number of gluons. We have presented a method to compute the modified function $E(z, p, \varepsilon)$. The modified function is given as a linear combination of $(n-3)$ ! basis amplitudes. Each term in this linear combination is gaugeinvariant and has the correct transformation properties under PSL $(2, \mathbb{C})$-transformations. We then moved on towards amplitudes not satisfying the BCJ-relations. This concerns QCD amplitudes with two or more quark-antiquark pairs. These amplitudes require in addition a generalisation of the Parke-Taylor factors. We derived a sufficient and necessary condition for such a generalisation and worked out the simplest case of the QCD tree-level four-point amplitude with two quarkantiquark pairs explicitly. Finding the correct generalised Parke-Taylor factors for all QCD multiquark primitive amplitudes is a direction for future research.

\section{A Feynman rules}

In this appendix we give a list of the colour ordered Feynman rules. They are obtained from the standard Feynman rules by extracting from each formula the coupling constant and the colour part. The propagators for quark and gluon particles are given by

$$
\begin{aligned}
& \longleftarrow=i \frac{\not k+m}{k^{2}-m^{2}}, \\
& \text { QQeee }=\frac{-i g^{\mu \nu}}{k^{2}} .
\end{aligned}
$$

The colour ordered Feynman rules for the three-gluon and the four-gluon vertices are

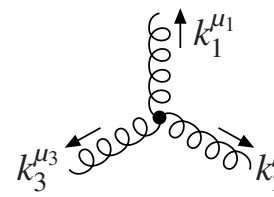

$$
=i\left[g^{\mu_{1} \mu_{2}}\left(k_{1}^{\mu_{3}}-k_{2}^{\mu_{3}}\right)+g^{\mu_{2} \mu_{3}}\left(k_{2}^{\mu_{1}}-k_{3}^{\mu_{1}}\right)+g^{\mu_{3} \mu_{1}}\left(k_{3}^{\mu_{2}}-k_{1}^{\mu_{2}}\right)\right],
$$

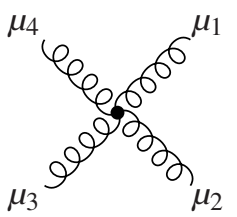

$$
=i\left[2 g^{\mu_{1} \mu_{3}} g^{\mu_{2} \mu_{4}}-g^{\mu_{1} \mu_{2}} g^{\mu_{3} \mu_{4}}-g^{\mu_{1} \mu_{4}} g^{\mu_{2} \mu_{3}}\right] .
$$


The Feynman rule for the quark-gluon vertex is given by
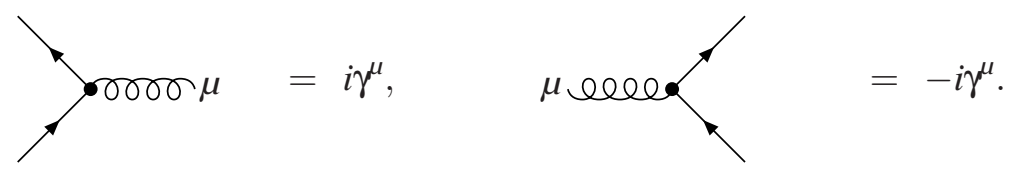

Open Access. This article is distributed under the terms of the Creative Commons Attribution License (CC-BY 4.0), which permits any use, distribution and reproduction in any medium, provided the original author(s) and source are credited.

\section{References}

[1] F. Cachazo, S. He and E.Y. Yuan, Scattering in Three Dimensions from Rational Maps, JHEP 10 (2013) 141 [arXiv:1306.2962] [inSPIRE].

[2] F. Cachazo, S. He and E.Y. Yuan, Scattering equations and Kawai-Lewellen-Tye orthogonality, Phys. Rev. D 90 (2014) 065001 [arXiv:1306.6575] [INSPIRE].

[3] F. Cachazo, S. He and E.Y. Yuan, Scattering of Massless Particles in Arbitrary Dimensions, Phys. Rev. Lett. 113 (2014) 171601 [arXiv:1307.2199] [INSPIRE].

[4] F. Cachazo, S. He and E.Y. Yuan, Scattering of Massless Particles: Scalars, Gluons and Gravitons, JHEP 07 (2014) 033 [arXiv:1309.0885] [INSPIRE].

[5] F. Cachazo, S. He and E.Y. Yuan, Einstein- Yang-Mills Scattering Amplitudes From Scattering Equations, JHEP 01 (2015) 121 [arXiv: 1409.8256 ] [INSPIRE].

[6] F. Cachazo, S. He and E.Y. Yuan, Scattering Equations and Matrices: From Einstein To Yang-Mills, DBI and NLSM, arXiv: 1412.3479 [INSPIRE].

[7] L. Dolan and P. Goddard, Proof of the Formula of Cachazo, He and Yuan for Yang-Mills Tree Amplitudes in Arbitrary Dimension, JHEP 05 (2014) 010 [arXiv : 1311. 5200] [INSPIRE].

[8] R. Britto, F. Cachazo, B. Feng and E. Witten, Direct proof of tree-level recursion relation in Yang-Mills theory, Phys. Rev. Lett. 94 (2005) 181602 [hep-th/0501052] [inSPIRE].

[9] L. Dolan and P. Goddard, The Polynomial Form of the Scattering Equations, JHEP 07 (2014) 029 [arXiv: 1402.7374$]$ [INSPIRE].

[10] Y.-H. He, C. Matti and C. Sun, The Scattering Variety, JHEP 10 (2014) 135 [arXiv:1403.6833] [INSPIRE].

[11] R. Monteiro and D. O'Connell, The Kinematic Algebras from the Scattering Equations, JHEP 03 (2014) 110 [arXiv: 1311.1151] [INSPIRE].

[12] C. Kalousios, Massless scattering at special kinematics as Jacobi polynomials, J. Phys. A 47 (2014) 215402 [arXiv:1312.7743] [inSPIRE].

[13] S. Weinzierl, On the solutions of the scattering equations, JHEP 04 (2014) 092 [arXiv:1402.2516] [INSPIRE].

[14] C.S. Lam, Permutation Symmetry of the Scattering Equations, Phys. Rev. D 91 (2015) 045019 [arXiv: 1410.8184$]$ [INSPIRE]. 
[15] S.G. Naculich, Scattering equations and BCJ relations for gauge and gravitational amplitudes with massive scalar particles, JHEP 09 (2014) 029 [arXiv: 1407. 7836] [INSPIRE].

[16] L. Mason and D. Skinner, Ambitwistor strings and the scattering equations, JHEP 07 (2014) 048 [arXiv:1311.2564] [INSPIRE].

[17] N. Berkovits, Infinite Tension Limit of the Pure Spinor Superstring, JHEP 03 (2014) 017 [arXiv:1311.4156] [INSPIRE].

[18] H. Gomez and E.Y. Yuan, N-point tree-level scattering amplitude in the new Berkovits'string, JHEP 04 (2014) 046 [arXiv: 1312.5485] [inSPIRE].

[19] T. Adamo, E. Casali and D. Skinner, Ambitwistor strings and the scattering equations at one loop, JHEP 04 (2014) 104 [arXiv: 1312 . 3828] [inSPIRE].

[20] Y. Geyer, A.E. Lipstein and L.J. Mason, Ambitwistor Strings in Four Dimensions, Phys. Rev. Lett. 113 (2014) 081602 [arXiv:1404 .6219] [INSPIRE].

[21] E. Casali and P. Tourkine, Infrared behaviour of the one-loop scattering equations and supergravity integrands, arXiv:1412.3787 [iNSPIRE].

[22] B.U.W. Schwab and A. Volovich, Subleading Soft Theorem in Arbitrary Dimensions from Scattering Equations, Phys. Rev. Lett. 113 (2014) 101601 [arXiv:1404.7749] [inSPIRE].

[23] N. Afkhami-Jeddi, Soft Graviton Theorem in Arbitrary Dimensions, arXiv:1405.3533 [INSPIRE].

[24] M. Zlotnikov, Sub-sub-leading soft-graviton theorem in arbitrary dimension, JHEP 10 (2014) 148 [arXiv:1407.5936] [inSPIRE].

[25] C. Kalousios and F. Rojas, Next to subleading soft-graviton theorem in arbitrary dimensions, JHEP 01 (2015) 107 [arXiv: 1407.5982$]$ [inSPIRE].

[26] C.D. White, Diagrammatic insights into next-to-soft corrections, Phys. Lett. B 737 (2014) 216 [arXiv:1406.7184] [inSPIRE].

[27] R. Monteiro, D. O'Connell and C.D. White, Black holes and the double copy, JHEP 12 (2014) 056 [arXiv:1410.0239] [inSPIRE].

[28] R. Roiban, M. Spradlin and A. Volovich, On the tree level S matrix of Yang-Mills theory, Phys. Rev. D 70 (2004) 026009 [hep-th / 0403190 ] [inSPIRE].

[29] N.E.J. Bjerrum-Bohr, P.H. Damgaard, P. Tourkine and P. Vanhove, Scattering Equations and String Theory Amplitudes, Phys. Rev. D 90 (2014) 106002 [arXiv: 1403.4553] [inSPIRE].

[30] R. Kleiss and H. Kuijf, Multi - Gluon Cross-sections and Five Jet Production at Hadron Colliders, Nucl. Phys. B 312 (1989) 616 [inSPIRE].

[31] Z. Bern, J.J.M. Carrasco and H. Johansson, New Relations for Gauge-Theory Amplitudes, Phys. Rev. D 78 (2008) 085011 [arXiv: 0805. 3993] [inSPIRE].

[32] F.A. Berends and W.T. Giele, Recursive Calculations for Processes with n Gluons, Nucl. Phys. B 306 (1988) 759 [inSPIRE].

[33] M.L. Mangano and S.J. Parke, Multiparton amplitudes in gauge theories, Phys. Rept. 200 (1991) 301 [hep-th/0509223] [iNSPIRE].

[34] C. Reuschle and S. Weinzierl, Decomposition of one-loop QCD amplitudes into primitive amplitudes based on shuffle relations, Phys. Rev. D 88 (2013) 105020 [arXiv: 1310.0413] [inSPIRE].

[35] B. Feng, R. Huang and Y. Jia, Gauge Amplitude Identities by On-shell Recursion Relation in S-matrix Program, Phys. Lett. B 695 (2011) 350 [arXiv: 1004 . 3417] [inSPIRE]. 
[36] N.E.J. Bjerrum-Bohr, P.H. Damgaard and P. Vanhove, Minimal Basis for Gauge Theory Amplitudes, Phys. Rev. Lett. 103 (2009) 161602 [arXiv:0907.1425] [inSPIRE].

[37] S. Stieberger, Open and Closed vs. Pure Open String Disk Amplitudes, arXiv:0907.2211 [INSPIRE].

[38] N. Arkani-Hamed and J. Kaplan, On Tree Amplitudes in Gauge Theory and Gravity, JHEP 04 (2008) 076 [arXiv: 0801.2385$]$ [INSPIRE].

[39] N. Arkani-Hamed, F. Cachazo and J. Kaplan, What is the Simplest Quantum Field Theory?, JHEP 09 (2010) 016 [arXiv: 0808.1446] [inSPIRE].

[40] C. Schwinn and S. Weinzierl, On-shell recursion relations for all Born QCD amplitudes, JHEP 04 (2007) 072 [hep-ph/0703021] [inSPIRE].

[41] C. Cheung, On-Shell Recursion Relations for Generic Theories, JHEP 03 (2010) 098 [arXiv: 0808.0504 ] [INSPIRE].

[42] Y. Jia, R. Huang and C.-Y. Liu, $U(1)$-decoupling, KK and BCJ relations in $\mathcal{N}=4$ SYM, Phys. Rev. D 82 (2010) 065001 [arXiv: 1005.1821] [inSPIRE].

[43] H. Kawai, D.C. Lewellen and S.H.H. Tye, A Relation Between Tree Amplitudes of Closed and Open Strings, Nucl. Phys. B 269 (1986) 1 [inSPIRE].

[44] N.E.J. Bjerrum-Bohr, P.H. Damgaard, B. Feng and T. Sondergaard, Gravity and Yang-Mills Amplitude Relations, Phys. Rev. D 82 (2010) 107702 [arXiv: 1005 . 4367] [INSPIRE].

[45] N.E.J. Bjerrum-Bohr, P.H. Damgaard, T. Sondergaard and P. Vanhove, The Momentum Kernel of Gauge and Gravity Theories, JHEP 01 (2011) 001 [arXiv: 1010.3933] [INSPIRE].

[46] A. Ben-Israel and T. Greville, Generalized Inverses: Theory and Applications, Springer, New York (2003).

[47] M. Koecher, Lineare Algebra und analytische Geometrie, Springer, Berlin (1997). 